\title{
FEATURES OF THE $\Sigma 5$ AND $\Sigma 9$ GRAIN BOUNDARIES MIGRATION IN BCC AND FCC METALS UNDER SHEAR LOADING - A MOLECULAR DYNAMICS STUDY
}

\author{
UDC 539.386
}

\author{
Andrey I. Dmitriev, Anton Yu. Nikonov \\ Institute of Strength Physics and Materials Science SB RAS, Tomsk, Russia \\ Tomsk State University, Tomsk, Russia
}

\begin{abstract}
Molecular dynamics simulation of metallic bicrystals has been carried out to investigate the behavior of the symmetrical tilt grain boundaries under shear loading. $\Sigma 5$ and $\Sigma 9$ grain boundaries in Ni and $\alpha$-Fe were analyzed. It is found that behavior of the defect depends not only on the structure of boundaries but also on the type of crystal lattice. In particular it is shown that under external stress the grain boundary (GB) behaves differently in the BCC and FCC metal. A comparison of the values of displacement of various types of $G B$ due to their migration caused by shear deformation is carried out. The results can help us to understand the features of the plastic deformation development in nanoscale polycrystals under shear loading.
\end{abstract}

Key Words: Molecular Dynamics, Symmetrical Tilt Grain Boundary, Shear Loading, Grain Boundary Migration, Non-equilibrium Structure

\section{INTRODUCTION}

Getting new knowledge about mechanisms of the plastic deformation development is one of the key challenges of modern materials science. This task has been the subject of intensive experimental and theoretical works. The results they achieve show that for polycrystalline materials the features of plastic deformation are determined not only by the loading conditions, but mainly by the characteristic dimensions of the grain structure. Thus, for metals with a relatively large grain size, the main mechanisms for the plastic deformation development are the motion of dislocations, which leads to the appearance of deformation localization bands. When decreasing the grain size and thus increasing the

Received May 12, 2017 / Accepted June 20, 2017

Corresponding author: Andrey I. Dmitriev

Institute of Strength Physics and Materials Science SB RAS, 634055, pr. Akademicheski 2/4, Tomsk, Russia.

E-mail:dmitr@ispms.ru 
total proportion of the grain boundaries, the grain boundary sliding becomes the dominant deformation mechanism [1-4]. As a result, the materials consisting of very small crystal grains are known to show some particular behavior. A typical example is the superplastic deformation of metals under tensile stress, which elongates up to several hundred percent without fracture [5]. That high deformation of bulk materials is considered to be controlled by the slip of grains at the boundary in the rearrangement of the grain configuration. However, this mechanism seems to be much more complicated; new data are required with respect to atomic transfer both inside and outside the grains. It is shown in a number of studies, that it is possible to form interfacial non-equilibrium structures by shear deformation that exists only during the stage of the active loading $[6,7]$. At the same time, due to a great variety and transience of the processes as well as the complexity of the experimental observation of deformation mechanisms realized near the interfaces, the methods of computer simulation can be considered as a useful tool [8-11]. In particular, molecular dynamics (MD) simulation appears to be a good method to follow the atomic ensemble evolution under such a loading. The advantage of this method is in the possibility to investigate elementary atomic mechanisms realized near the interfaces and then to use the results for modeling at higher scales as well as for interpreting the experimental data.

Earlier [10] it has been shown by means of MD simulation that the external shear deformation leads to restructuring the crystal lattice near the grain boundaries. Such a restructuration, in its turn, may result in a preferential growth of only one grain on account of the other neighboring ones. The results in the cited paper are obtained on a sample of the copper containing symmetrical tilt grain boundary $\Sigma 5$ (210)[001]. Later, similar studies have been done on copper bicrystals containing tilt grain boundaries different from $\Sigma 5$ (210)[001] ones [10]. Studies have shown that the behavior of grain boundaries such as, for example $\Sigma 9(1 \overline{2} 2)[011]$, under shear deformation may significantly differ from the behavior of the $\Sigma 5$ grain boundary. Thus, if the Y-coordinate of $\Sigma 5$ GB due to shear deformation changes in the direction perpendicular to applied loading then $\Sigma 9$ GB does not migrate in the bulk of the material and shear deformation provides the formation of structural changes along the plane of the defect.

The aim of this study is to check the generality of the observed grain boundary sliding mechanism by modeling the behavior of these defects under shear loading conditions for nickel and $\alpha$-iron bicrystal samples.

\section{NUMERICAL MODEL}

Investigations are carried out within the framework of the conventional molecular dynamics method using the Large-scale Atomic/Molecular Massively Parallel Simulator (LAMMPS) software [13, 14]. The visualization of MD simulations data and structure analysis is carried out using the open visualization tool OVITO [15]. The behavior of bicrystals with the initial face-centered-cubic (fcc) and body-centered-cubic (bcc) structures is investigated by the examples of $\mathrm{Ni}$ and $\alpha$-Fe crystallites. Within the numerical simulation of grain boundary behavior, there is a problem of generating the initial structure of the defect. The peculiarity of the GBs in the general form is their non-periodicity. Since only a fragment of the sample is modeled within the framework of molecular dynamics method, which is later repeated due to the use of periodic boundary conditions, it is necessary to consider a 
sufficiently extended fragment to generate GB of a general form. Therefore, the special type grain boundaries that have a periodic structure are most often used with the MD method.

In both considered cases, the modeled specimens are in the form of rectangular fragments and contain a planar structural defect such as a high-angle tilt grain boundary oriented as shown in Fig. 1. Tilt grain boundaries $\Sigma 5(210)[001]$ and $\Sigma 9(1 \overline{2} 2)[011]$ are obtained by means of the Coincidence Site Lattice (CSL) principle. The algorithm to design the initial structure is described in details in [16] and it includes the following steps. Firstly, the grain orientation in space is that external axes $\mathrm{X}, \mathrm{Y}$ and $\mathrm{Z}$ correspond to the crystallographic directions determined by the type of defect. Then, the second grain is generated by mirroring the first one in plane XoZ, which then becomes the plane of the defect. The last step involves the relaxation of the resulting sample.

The modeled structure is located between the two loaded layers and subjected to a sliding loading with constant velocities $(\mathrm{V})+20$ and $-20 \mathrm{~m} / \mathrm{s}$ applied to the upper and lower layers, respectively, as shown schematically in Fig. 1. Thus, the resulting shear loading rate is $40 \mathrm{~m} / \mathrm{s}$. The sample with an initial temperature of $200 \mathrm{~K}$ is simulated. A certain temperature is achieved by using the velocity rescaling method during the MD simulation process from the energy balance described by Eq. (1):

$$
\frac{1}{N} \sum_{i}^{N} \frac{m_{i} v_{i}^{2}}{2}=\frac{3}{2} k_{b} T
$$

where $N$ is the atom number, $k_{b}$ is the Boltzmann constant, $m_{i}$ and $v_{i}$ are the $i^{\text {th }}$ atom mass and velocity, respectively.

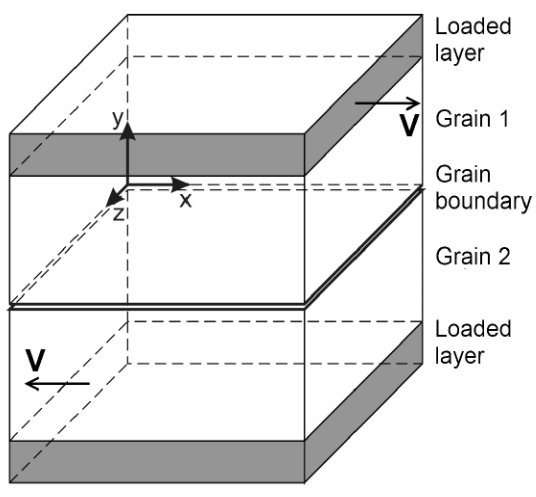

Fig. 1 Schematic representation of the simulated sample

A periodic boundary condition is prescribed in the $\mathrm{X}$ - and Z-directions of the specimen to reduce the simulation scale effect. Atomic interaction is described in the framework of the embedded atom method [17]. The total number of atoms is about of 100000. The modeled sample is considered as an NVE ensemble maintaining the number of particles $N$, and occupying volume $V$ and the energy of system $E$. The equations of motion are integrated on the basis of the Velocity-Verlet algorithm with a time step $\Delta t$ of $1 \times 10^{-15} \mathrm{~s}$. 


\section{SHEAR LOADING OF NI BICRYSTAL}

Initially, the modeling of grain boundaries behavior in a nickel sample under conditions of shear deformation is carried out. The calculated values of the specific energy of the considered plane defects are as follows: $\mathrm{E}_{\Sigma 5}=2,290 \mathrm{~J} / \mathrm{m}^{2}, \mathrm{E}_{\Sigma 9}=1,309 \mathrm{~J} / \mathrm{m}^{2}$. Firstly, we investigate the behavior of nickel sample containing the grain boundary $\Sigma 5$. Since the atomic lattice of $\mathrm{Ni}$ is fcc, i.e. similar to that of $\mathrm{Cu}$, we expect a similarity in a response of $\mathrm{Ni}$ crystallite with identical GB. The projection of atoms on the plane XoY of the fragment with initial structure containing a planar defect is shown in Fig. 2a. Black color indicates the atoms lying in the plane of the defect, dark gray color denotes atoms arranged initially in a plane perpendicular to that of the grain boundary and used for the deformation distribution visualization along the specimen. The results of modeling the behavior of grain boundaries $\Sigma 5$ in nickel sample show that under conditions of shear deformation the relative sliding of interacting grains is accompanied by displacement of boundary position in the direction perpendicular to the applied loading. The resulting structure of the modeled specimen after 200000 steps of sliding is shown in Fig. 2b. The magnitude of this displacement is by a factor of 1.5 greater than the relative slip between the grains very much alike it was observed previously for the same GB in Cu bicrystal [10,14]. It should also be noted that the configuration of the atomic lattice near the plane of the defect retains a regular arrangement of atoms throughout the process of moving the grain boundary so that no non-equilibrium structures are formed.

(a)

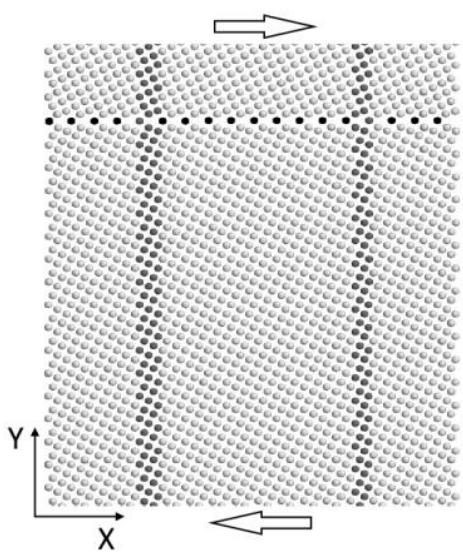

(b)

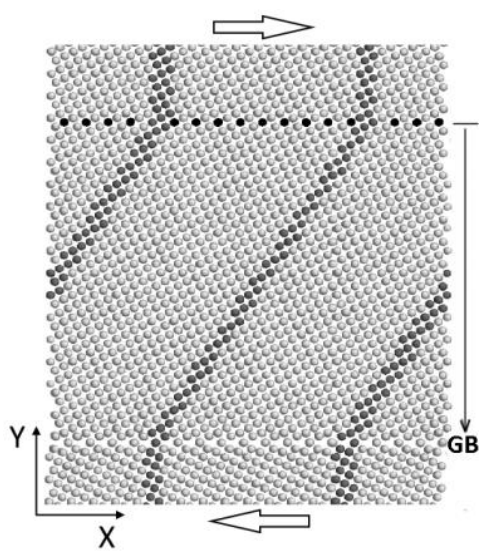

Fig. 2 The projection of atomic structure near the grain boundary $\Sigma 5$ on the plane XoY at different moments of time a) $t=0 p s ; b) t=200 p s$

The next stage of our research is to model the behavior of $\mathrm{Ni}$ sample containing a grain boundary $\Sigma 9$. The resulting equilibrium atomic configuration near the plane of the defect is shown in Fig. 3a. A peculiarity of the $\Sigma 9$ grain boundary structure is its asymmetric character with respect to the defect plane [18]. According to the results of simulation, the shear loading has never been able to move such a boundary in the direction perpendicular to the defect plane. Under shear loading, only a relative slippage occurs between the grains along the $\mathrm{X}$ axis which is accompanied by curving the defect plane. The latter is confirmed by the 
resulting distribution of marked atoms initially arranged perpendicular to the GB plane. This is well seen in Fig. 3b, where the structure of the central part of the sample is shown at time $t$ $=100 \mathrm{ps}$.

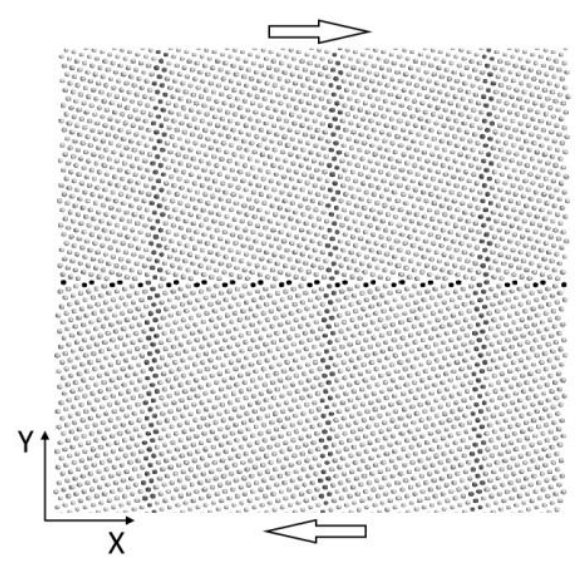

(a)

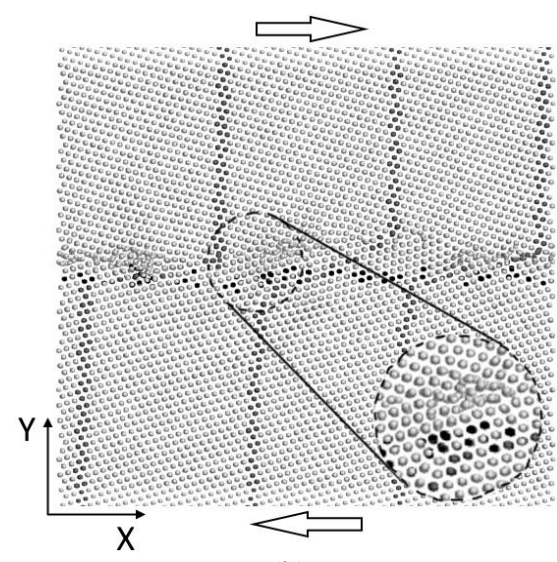

(b)

Fig. 3 The projection of atomic structure near the grain boundary $\Sigma 9$ on the plane XoY at different moments of time a) $t=0 p s ; b) t=100 p s$

To analyze the features of the structural location of each atom during shear loading simulation the common neighbor analysis (CNA) is used [19]. According to CNA, a single hexagonal-close-packed (hcp) coordinated-layer means a coherent twin boundary while two adjacent hcp-coordinated-layers indicate an intrinsic stacking fault. Fig. 4a shows the projection of the central fragment of the structure after 100000 integration steps.

In Fig. 4 bright gray dots mark atoms belonging to the defect-free fcc lattice. Atoms defined as those of local hcp structure are marked by large dark gray circles. Black dots

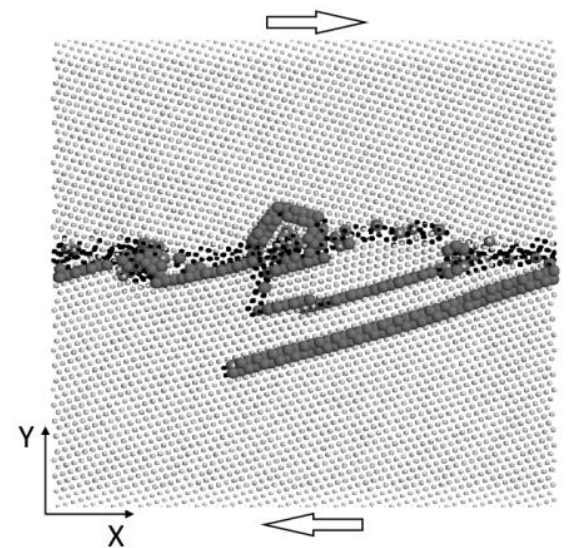

(a)

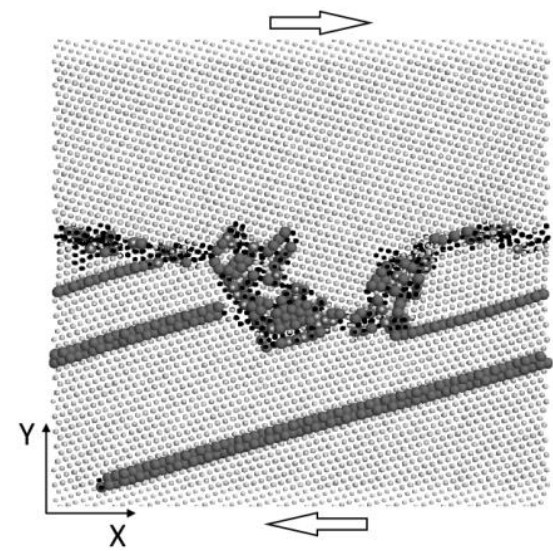

(b)

Fig. 4 Structure (projection on XoY plane) near the GB $\Sigma 9$ at different moments of time (a) $t=100 p s$ and (b) $t=160 p s$. Atoms are colored according to CNA value 
denote atoms whose local structural allocation differs from fcc, bcc and hcp. Such atoms are located near the grain boundaries and other structural defects and can be used to identify the position of the border between two grains. Further loading of bicrystal with GB $\Sigma 9$ leads to the formation of separate segments crystallographically misoriented with respect to each other as those seen in Fig. 4b. Again, no non-equilibrium structure formation is observed.

Comparing the behavior of copper and nickel bicrystals containing $\Sigma 5$ and $\Sigma 9$ symmetrical tilt grain boundaries, we observe a good qualitative agreement between the shear deformation simulation results as was expected due to identical atomic lattice of both metals. Therefore, one can expect a similar behavior for other metals with face-centered cubic lattice under shear loading.

\section{THE BEHAVIOR OF $\Sigma 5$ AND $\Sigma 9$ GBS IN IRON SAMPLE}

To verify the generality of the revealed regularities of the behavior of grain boundaries on other types of atomic lattices, shear deformation of metal with bcc structure was carried out. Specimens of $\alpha$-iron with symmetrical tilt grain boundaries $\Sigma 5$ (120)[001] and $\Sigma 9$ (114)[110] were simulated. The calculated values of the specific energy of the considered plane defects were as follows: $\mathrm{E}_{\Sigma 5}=1,122 \mathrm{~J} / \mathrm{m}^{2}, \mathrm{E}_{\Sigma 9}=1,309 \mathrm{~J} / \mathrm{m}^{2}$, respectively. Firstly, we investigated the behavior of $\alpha$-iron sample containing the grain boundary $\Sigma 5$. The algorithm similar to that described above was used to generate the initial structure. Firstly, the grain orientation in space was specified in such a way that external axes $\mathrm{X}, \mathrm{Y}$ and $\mathrm{Z}$ corresponded to the crystallographic orientations determined by the type of the defect. Then the second grain was generated by mirroring the first one in plane XoZ, which then became the plane of the defect. The next step included finding the minimum of potential energy by relative displacement of grains along the plane of the defect. Shift vector for grain boundary $\Sigma 5$ was $\mathbf{t}=(1,28 ; 0 ; 0)$. The last step contained the relaxation of the resulting structure. The projection on XoY plane of atomic configuration after relaxation of $\alpha$-iron specimen containing the grain boundary $\Sigma 5$ (120)[001] is shown in Fig. 5. The loading as well as boundary conditions were identical to those used for shear deformation of nickel bicrystal.

The results show that the response of the simulated crystallite containing the grain boundary $\Sigma 5$ differs from those previously obtained on $\mathrm{Ni}$ and $\mathrm{Cu}$ bicrystals. According to the simulation data, the response of the specimen to external shear loading can be divided into three stages as follows. Initially, the atomic lattice is deformed elastically outside of the plane of the defect (stage I). This is confirmed by a spatial distribution of atoms marked by dark gray color shown in Fig. 6a. Next stage includes the formation of crystal structure that is different from the initial one and located near to the original position of GB. In accordance with the simulation results, the thickness of this layer in our example reaches of 4-5 inter-planar distances. In Fig. $6 \mathrm{~b}$ the border of this layer is highlighted by dashed lines. Due to action of periodic boundary conditions along $\mathrm{X}$ and $\mathrm{Z}$ axes this structure can be associated with a thin in-plane defect. 


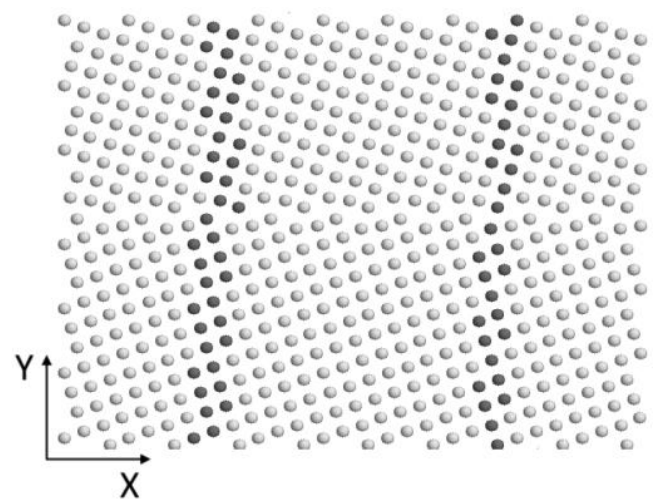

Fig. 5 The initial structure (projection on XY plane) of $\alpha$-iron sample with GB $\Sigma 5$

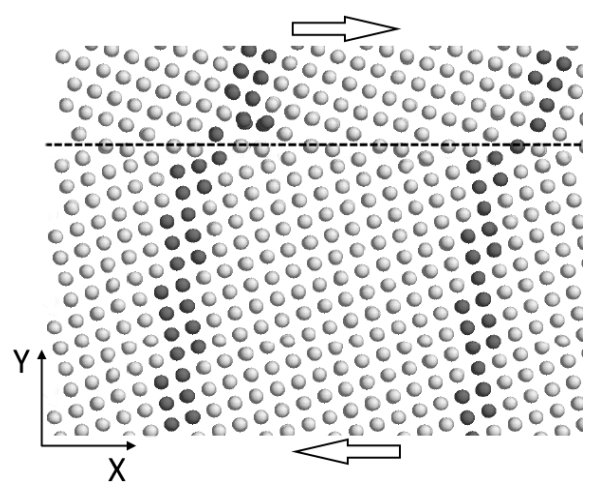

(a)

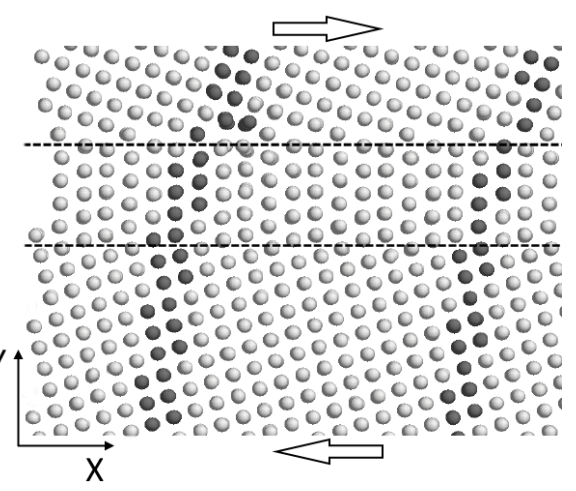

(b)

Fig. 6 The fragment of the structure (projection on the plane XoY) of atomic configuration near the grain boundary $\Sigma 5$ at different moments of time (a) after 40 ps and (b) after 60 ps of shear loading for $\alpha$-Fe bicrystal

The observed crystal structure is not stable because its further deformation leads to restructuring. This is accompanied by a mismatch of the ordered structure near the grain boundary, which is analogous to that observed in a copper sample with a grain boundary $\Sigma 5$ at high sample temperatures or with an imperfect initial boundary structure $[10,14]$. After that the grain boundary starts moving in the direction perpendicular to the applied external loading (stage III). Analysis of the structure at different moments of time shows that despite the disordered structure near the defect, the boundary moves uniformly with constant relative velocity, which is 1,2 times higher than the rate of external loading. The mentioned above stages are clearly seen on the time dependence of the grain boundary position along Y direction shown in Fig. 7a. The stage II was associated with a sharp change in the GB position, which occurred due to restructuring of the non-stable layer that took place between $45 \mathrm{ps}$ and $70 \mathrm{ps}$ of the loading time. The founded lifetime of the stage II (about 25 ps) is too short to be identified experimentally but we expect that it should be sensitive to the loading conditions. 
The resulting configuration of the structure of the sample that contains grain boundaries after 200000 integration steps is shown in Fig. 7b. The spatial distribution of marked atoms confirms monotonically migration of the grain boundary at the stage III. The disordered structure near the GB is also well seen.

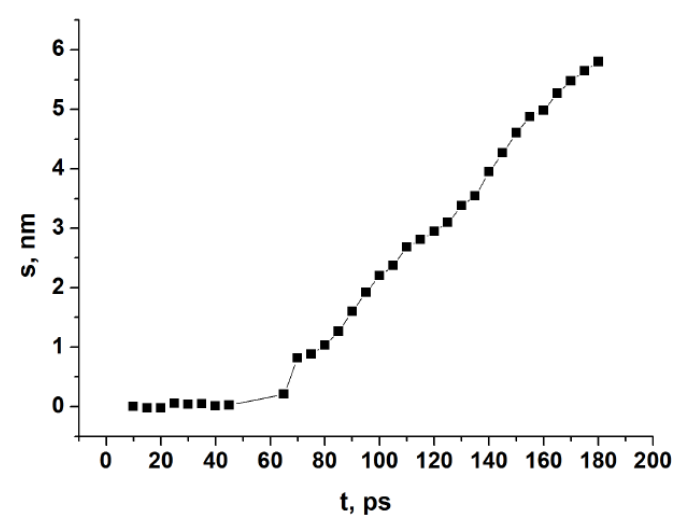

(a)

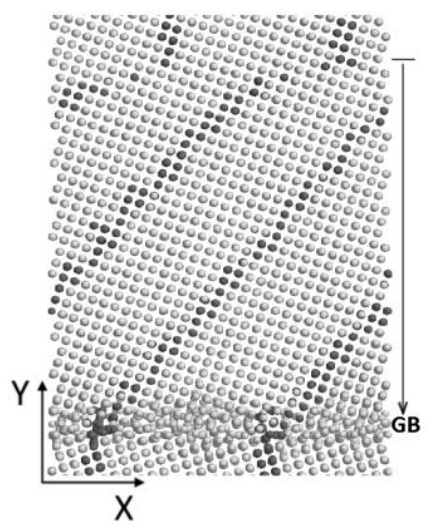

(b)

Fig. 7 a) Time dependence of boundary displacement $s$ on the initial position;

b) The structure (projection of atoms on XoY plane) of $\alpha$-Fe specimen with the grain boundary $\Sigma 5$ after 200 ps of shear loading. The arrow shows the direction of GB migration during shear deformation at stage III

The next stage of our research was to model the behavior of $\Sigma 9$ grain boundary under shear deformation. To generate this type of symmetrical tilt GB, the vector of relative shift was as follows $\mathbf{t}=(10,17576 ; 0,5325 ; 1,04988)$. Fig. 8a shows the resulting structure of the bicrystal containing such a GB after relaxation of the sample. The simulation results show that the behavior of such a defect in $\alpha$-iron under shear deformation is similar

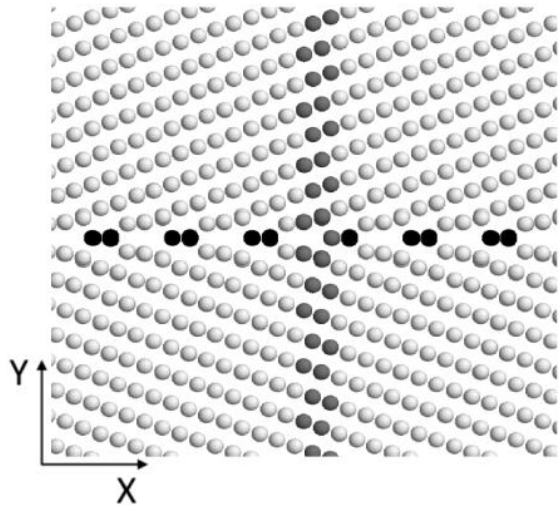

(a)

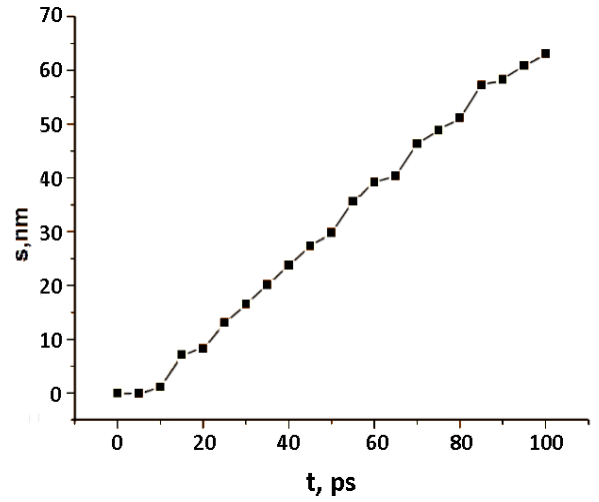

(b)

Fig. 8 a) The initial structure (projection of atoms on XoY plane) $\alpha$-Fe specimen with GB $\Sigma 9$; b) Time dependence of boundary displacement $s$ on the initial position 
to that of grain boundary $\Sigma 5(210)[001]$ in fcc metals. Due to the shear loading a synchronous restructuring of atomic lattice near the plane of the defect occurred that, in its turn, led to the grain boundary motion in the direction perpendicular to the applied loading. At that the structure of the defect is still regular. The simulation results show that under loading the $\Sigma 9$ grain boundary begins to move along $\mathrm{Y}$ axis with a practically constant velocity (Fig. 8b) which is defined by the shear loading rate. Thus, the estimations give us about $70 \mathrm{~m} / \mathrm{s}$ for the grain boundary migration velocity while the relative sliding velocity of two loaded layers is $40 \mathrm{~m} / \mathrm{s}$.

\section{CONCLUSIONS}

Using the MD simulations the features of the grain boundary sliding mechanism have been studied on the scale of individual atoms by the example of large angle symmetrical tilt grain boundaries $\Sigma 5$ and $\Sigma 9$ in bicrystals of $\mathrm{Ni}$ and $\alpha$-Fe. It is shown that for a certain orientation of the defect a grain boundary sliding under shear loading may be accompanied by rearrangement of the atomic configuration in the plane of the defect, which leads to an efficient movement of the position of GB in the direction perpendicular to applied loading. At that, the boundary displacement velocity can be several times higher than the shear loading rate. It is found that the dynamic properties of the boundary migration depend both on the features of GB structure and the type of crystal lattice. In particular, the "mobility" of grain boundary $\Sigma 5$ under shear deformation in fcc lattice disappears in the crystallite with bcc structure, and vice versa - "unmovable" grain boundary $\Sigma 9$ in fcc bicrystal begins to move (shift) in the direction perpendicular to the applied loading with changing the type of atomic lattice. Note that in this paper we study only an elementary grain boundary slip mechanism that is realized in pure bicrystals with periodic boundary conditions on the stage of active deformation. The influence of triple junctions and other possible sources of resistance on grains relative slip have not been taken into account. Nevertheless, these results can be used for a better understanding of the main features of plastic deformation development in nanoscale polycrystals.

The founded configurations of atoms in non-equilibrium state have a separate fundamental meaning because they show non-linearity and complexity of the behavior of atomic system under dynamic loading conditions. This result also looks very interesting from the practical point of view and hence it requires additional investigations in order to find the conditions conductive to stabilization of this state as it is planned to be done in our future works.

Acknowledgements: Investigations have been carried out with the financial support from Russian Science Foundation Grant No 17-19-01374.

\section{REFERENCES}

1. Bobylev, S.V., Morozov, N.F., Ovid'ko, I.A., 2010, Cooperative grain boundary sliding and migration process in nanocrystalline solids, Physical Review Letters, 105, pp. 055504/1-055504/4.

2. Masuda, H., Tobe, H., Sato, E., Sugino, Y., Ukai, S., 2016, Two-dimensional grain boundary sliding and mantle dislocation accommodation in ODS ferritic steel, Acta Materialia, 120, pp. 205-215. 
3. Ovid'ko, I.A., Sheinerman, A.G., 2016, Free surface effects on stress-driven grain boundary sliding and migration processes in nanocrystalline materials, Acta Materialia, 121, pp. 117-125.

4. Rupert, T.J., Gianola, D.S., Gan, Y., Hemker, K.J., 2009, Experimental observations of stress-driven grain boundary migration, Science, 326, pp. 1686-1690.

5. Toth, L.S., Gu, C., 2014, Ultrafine-grain metals by severe plastic deformation, Materials Characterization, 92, pp. 1-14.

6. Tyumentsev, A.N., Ditenberg, I.A., Korotaev, A.D., Denisov, K.I., 2013, Lattice curvature evolution in metal materials on meso- and nanostructural scales of plastic deformation, Physical Mesomechanics 16(4) pp. 319-334.

7. Tsukanov, A.A., Psakhie, S.G., 2016, Adhesion effects within the hard matter-soft matter interface: molecular dynamics, Facta Univesitatis Series Mechanical Engineering, 14(3), pp. 269-280.

8. Nikonov, A.Yu., Konovalenko, Iv.S., Dmitriev, A.I., 2016, Molecular dynamics study of lattice rearrangement under mechanically activated diffusion, Physical Mesomechanics, 19(1), pp. 77-85.

9. Dmitriev, A.I., Nikonov, A.Yu., Österle, W., 2017, Molecular dynamics sliding simulations of amorphous Ni, Ni-P and nanocrystalline Ni films, Computational Materials Science, 129, pp. 231-238.

10. Dmitriev, A.I. Nikonov, A.Yu., Psakhie, S.G., 2011, Atomistic mechanism of grain boundary sliding with the example of a large-angle boundary 55. Molecular dynamics calculation, Physical Mesomechanics, 14(1-2), pp. 24-31.

11. Psakhie, S.G., Popov, V.L., Shilko, E.V., Smolin, A.Yu., Dmitriev, A.I., 2009, Spectral analysis of the behavior and properties of solid surface layers. Nanotribospectroscopy, Physical Mesomechanics, 12(5-6), pp. 221-234.

12. Bondar, M.P., Psakhie, S.G., Dmitriev, A.I., Nikonov, A.Yu., 2013, On the conditions of strain localization and microstructure fragmentation under high-rate loading, Physical Mesomechanics, 16(3) pp. 191-199.

13. Plimpton, S. 1995, Fast parallel algorithms for short-range molecular dynamics, Journal of Computational Physics, 117(1), pp. 1-19.

14. Dmitriev, A.I., Nikonov, A.Yu., 2013, Simulation of the behavior of a 55 grain boundary under combined thermal and external shear loading, Technical Physics Letters, 39(8). pp. 709-712.

15. Stukowski, A., 2010, Visualization and analysis of atomistic simulation data with OVITO-the Open Visualization Tool, Modelling and Simulation in Materials Science and Engineering, 18, pp. 015012/1015012/7.

16. Suzuki, A., Mishin, Y., 2003, Atomistic modeling of point defects and diffusion in copper grain boundaries, Interface Science, 11(1) pp. 131-148.

17. Foiles, S.M. 1996, Embedded-atom and related methods for modeling metallic systems, MRS Bulletin, 21(2) pp. 24-28.

18. Van Swygenhoven, H., Farkas, D., Caro, A., 2000, Grain-boundary structures in polycrystalline metals at the nanoscale, Physical Review B, 62(2), pp. 831-838.

19. Honeycutt, J. and Andersen H., 1987, Molecular dynamics study of melting and freezing of small LennardJones clusters. Journal of Physical Chemistry, 91, pp. 4950-4963. 